\title{
SALAMANCA: COLABORANDO PARA NO PERDER LA PRESENCIA DE VERDE EN EL BARRIO
}

\author{
SALAMANCA: COLLABORATING \\ TO PRESERVE THE PRESENCE OF GREEN \\ IN THE NEIGHBORHOOD
}

\author{
ALEXANDER GALVEZ NIETO \\ Universidad Ricardo Palma \\ orcid.org/0000-0001-8526-0124
}

El artículo propone un estudio de la presencia de árboles urbanos en la subzona 03-Salamanca, como elemento atractivo del espacio público, desde un enfoque sostenible urbano. El área de estudio conforma parte de la zona 01 de Ate, considerada como pulmón del distrito perteneciente a la ciudad metropolitana de Lima, Perú. El desarrollo metodológico se basa en la determinación del volumen verde, su grado de mantenimiento y la generación de zonas de sombra. Los resultados se contrastan con las acciones municipales y de una organización civil defensora del arbolado urbano para establecer una estrategia verde colaborativa. La evaluación determinó un déficit del volumen verde y de zonas de sombra. Por ello, el apoyo ciudadano a través de sus asesorías, plantaciones y monitoreo de árboles constituye una oportunidad de aumento del atractivo del espacio público.

sostenibilidad, participación ciudadana, árboles urbanos, volumen verde, espacio atractivo, estrategia verde
Recibido: 13 de junio del 2020

Aprobado: 11 de noviembre del 2020

doi: https://doi.org/10.26439/limaq2021.n008.5552

The article proposes a study of the presence of urban trees in sub-zone 03 - Salamanca, as an attractive element of public space, from a sustainable urban approach. The study area is part of zone 01 of Ate, considered the lung of the district belonging to the metropolitan city of Lima, Peru. The methodology determines the green volume, its degree of maintenance and the generation of shadow areas. The results are compared with the municipal actions and a civil organization defending urban trees to establish a collaborative green strategy. The evaluation determined a deficit in green volume and shaded areas. For this reason, citizen support through its consultancies, plantations, and tree monitoring constitute an opportunity to increase the attractiveness of public space.

sustainability, citizen participation, urban trees, green volume, attractive space, green strategy 


\section{INTRODUCCIÓN}

Frente al cambio de la cobertura vegetal natural por los elementos construidos por el hombre en su beneficio (edificios, vías, áreas verdes, entre otros), se define una actitud de cuidar y conservar con el objeto de restablecer el balance en el metabolismo ambiental de la ciudad, para tener un buen vivir, lo que ha cambiado por la dominación destructiva irreversible realizada por el hombre (García Estrada et al., 2020). Así, con el tiempo, se acunó el término sostenibilidad, que actualmente tiene varias acepciones, pues el ambiente "es una dimensión mucho más compleja" (Valdivia-Loro, 2019) para limitar su acción solo a lo relacionado con la ecología. En el siglo xxI el concepto de atractivo es importante como parte de la dimensión cultural del desarrollo sostenible. La calidad del ambiente urbano es condición imprescindible para su capacidad de atracción y competitividad; por eso, las ciudades están preocupadas por destacar sus atractivos y atributos particulares, que los transforman mejor funcional y formalmente (Shigyo, 2017). Esta mejora de la calidad de vida urbana se logrará a través del espacio público (Daniel, 2019). Boff (como se citó en García Estrada et al., 2020) señala que la falta de socialización en espacios públicos ha provocado descuido y una carencia de identidad en estos.

En este lineamiento, "la presencia de verde es fundamental para aumentar el atractivo del espacio público" (Agencia de Ecología Urbana de Barcelona, 2011). Renaturalizar las ciudades, es decir, incrementar la vegetación, regenerará sosteniblemente los barrios (Del Caz-Enjuto, 2017). La habilitación o transformación de espacios públicos y zonas verdes en lugares seguros, cómodos y atractivos para vivir y trabajar garantizarán un traslado a pie o en bicicleta por la ciudad, así como la mejora de la calidad de vida de los habitantes de las ciudades. La presencia de una determinada cantidad de árboles urbanos puede garantizar una buena percepción visual de verde urbano, disminuir el impacto propio de los tejidos urbanos, mejorando el aspecto visual urbano y creando espacios vitales y dinámicos.

Estas acciones encaminadas hacia la sostenibilidad urbana se relacionan con la gestión en sus diferentes escalas políticas y espaciales, desde la global hasta la local (Pérez-Medina y López-Falfán, 2015). En el ámbito local es posible concretar dichas acciones a través de la planeación, la administración, el marco normativo y su monitoreo, así como por el rol de los diferentes grupos sociales e individuos que en conjunto construyen una gobernanza efectiva, en función del máximo beneficio para la ciudad (Reyna Valencia, 2017; Ruiz et al., 2016; Hansmann et al., 2016). Por ejemplo, en el Reino Unido, organizaciones tanto del sector público como del privado se unieron para formar Trees \& Design Action Group, el cual presentó en el 2012 una guía de principios y referencias para que los árboles puedan cumplir sus beneficios ambientales y sociales, y crear condiciones de éxito económico.

Ciudades como Valladolid (España), Liverpool (Reino Unido) y Esmirna (Turquía) han establecido soluciones basadas en la naturaleza (nature-based solutions, NbS), con el objetivo de "abordar los desafíos urbanos utilizando 
la naturaleza", incluyendo el compromiso de los ciudadanos y las actividades educativas (CARTIF, 2018). Se espera contribuir con las ciudades haciéndolas más atractivas, competitivas e inclusivas. En el caso de Valladolid, el logro es conectar las áreas verdes y complementar con acciones sociales y de apoyo a la economía verde, implicando a la población civil y organizaciones (Villazán y Huertas, 2019).

A pesar de todas estas iniciativas actuales, aún muchas ciudades presentan un deterioro del arbolado urbano debido a la interacción de factores bióticos, como el cambio climático, contaminación, estrés térmico, etcétera, así como a factores abióticos, como el deficiente establecimiento de árboles en el suelo urbano, cortes indebidos, etcétera (Restrepo et al., 2015). Aún falta una visión holística sobre el mantenimiento y cuidado de las especies arbóreas y arbustivas existentes, y espacios adecuados para la plantación (Gálvez Nieto, 2019).

Para los fines de la investigación, se adoptó el concepto de Valdivia-Loro (2019) que considera al ambiente como una dimensión compleja que abarca variables múltiples como lo social, ambiental, económico, además del atractivo, lo político, religioso, ético, entre otros. Y desde ese enfoque, desde la variable de atracción, se asume lo señalado por la Agencia de Ecología Urbana de Barcelona (2011) con relación a la percepción del volumen verde, indicador que influye en la percepción de las personas como elemento atractivo del espacio público.

En relación con esta situación, la subzona 03 de la zona 01 del distrito de Ate es un área consolidada y acogedora donde predominan las urbanizaciones, cuenta con accesibilidad a distritos importantes de la metrópoli: Santiago de Surco, San Luis y San Borja (Municipalidad de Ate, 2003), y posee la mayor cantidad de parques del distrito, en buen estado de conservación, razón por la cual pertenece al pulmón del distrito de Ate (Municipalidad de Ate, 2003). Sin embargo, dicha zona experimenta un cambio de morfología urbana por la sustitución de residencias unifamiliares por edificios multifamiliares, lo que incrementa rápidamente la cantidad de población (Galvez Nieto, 2019) y reduce la presencia del arbolado urbano que permanece solo en los parques vecinales, siendo retirado de las bermas de las calles. El manejo municipal del arbolado urbano se basa únicamente en los procesos de mantenimiento de las especies arbóreas, de las especies arbustivas y las superficies de los parques, a través del riego, corte del pasto y poda de árboles. Ante esto, ¿el área de Salamanca es una zona "verde" con suficiente presencia de verde?

\section{MATERIAL Y MÉTODOS}

La población de estudio está constituida por todos los árboles urbanos de la subzona 03-Salamanca, ubicada en la zona 01 del distrito de Ate, Lima, Perú (véase la figura 1). Para la selección de la muestra, se aplicó el método de muestreo no probabilístico de tipo intencional, por la representatividad que tienen estos espacios en la memoria de sus habitantes y su accesibilidad. Se elegirán los árboles del parque Fuerza Aérea del Perú y de los ocho tramos de la avenida Los Paracas. 
Figura 1

Subzona

03-Salamanca de la zona 01 del distrito de Ate

Elaboración propia

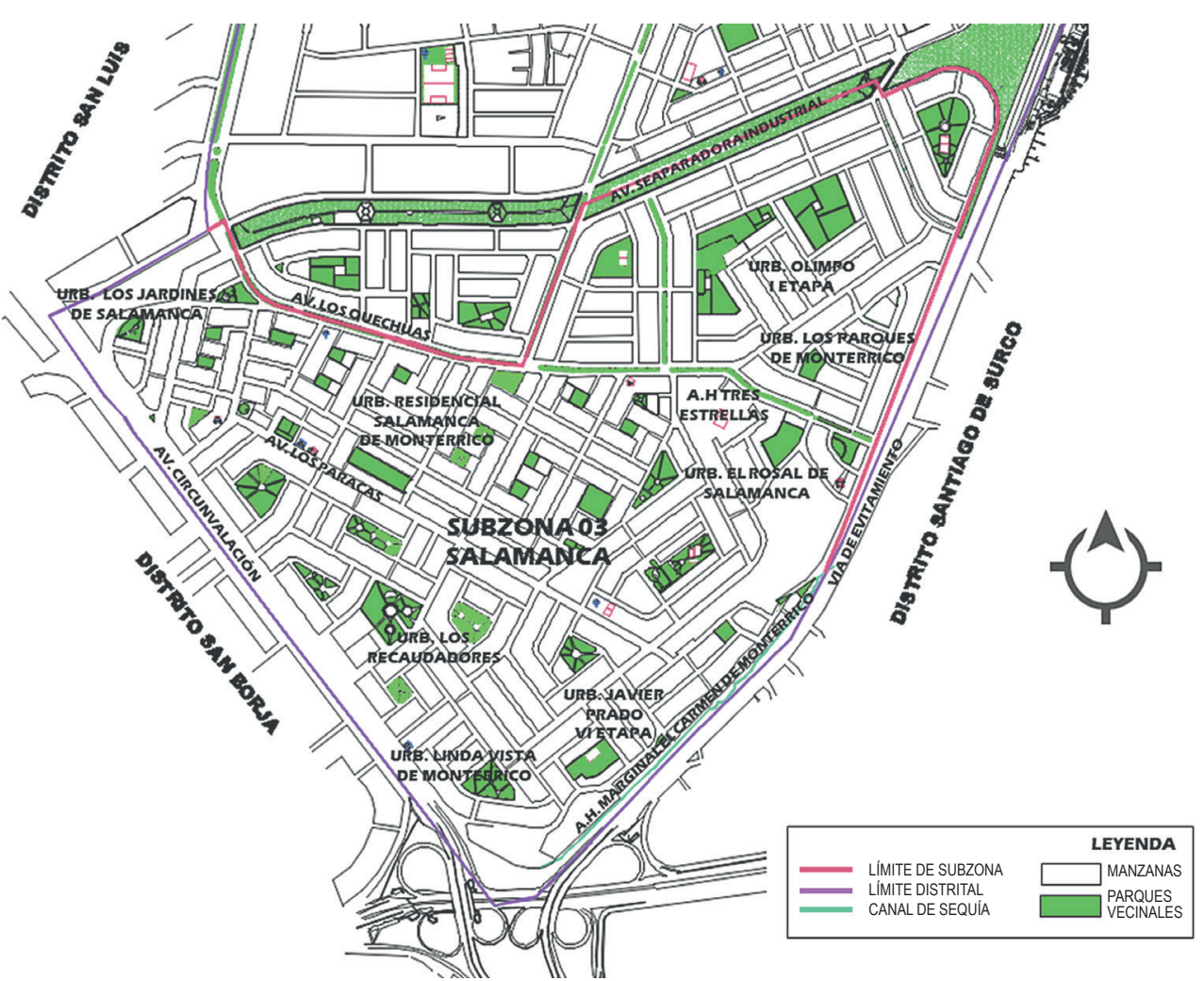

La propuesta está dirigida hacia la medición de la presencia de verde, por lo que se consideró la proporción del volumen verde según la Agencia de Ecología Urbana de Barcelona (2011) y la dotación de sombra por árboles (Mínguez et al., 2013). La investigación pretende poner de relieve cómo una cantidad determinada de árboles urbanos con un buen mantenimiento puede contribuir a la conversión de los espacios verdes urbanos en elementos atractivos y confortables de la ciudad. Los resultados se contrastaron con los principios de plantación/protección de árboles propuestos por Trees \& Design Action Group (2012) y las acciones municipales y de la asociación ambiental Salamanca Verde, una organización civil defensora del arbolado urbano.

En primer lugar, se analizaron las características del arbolado urbano existente y su relación con el tejido urbano de la subzona 03-Salamanca. Por consiguiente, los resultados se organizaron con los siguientes parámetros: las características de los árboles urbanos existentes, las restricciones físicas que presenta el espacio urbano y la identificación de la proporción del volumen verde. Para ello, se emplearon diversas técnicas de recolección de datos. Se empezó a recolectar información de instituciones públicas referida a las especies arbóreas del arbolado urbano, así como a los lineamientos sobre la 
plantación y mantenimiento de las áreas verdes de Lima Metropolitana y del distrito de Ate. Para las observaciones y mediciones del arbolado urbano, se elaboró un registro fotográfico. Solo se consideraron los árboles en etapa adulta con más de dos tercios de su ramificación, por poseer todos los atributos morfológicos totalmente desarrollados.

Además, se realizaron cálculos de parámetros sobre el volumen de árboles y el índice biótico del suelo, de acuerdo con la variable de espacios verdes y biodiversidad usada por la Agencia de Ecología Urbana de Barcelona (2011) en su Certificación del Urbanismo Ecosistémico. Se elaboraron mapas del parque y la avenida seleccionados para determinar la proporción urbana y las restricciones del sitio.

Finalmente, se procedió a establecer una relación entre el estado del arbolado urbano y las acciones de la gestión municipal y de Salamanca Verde.

\section{RESULTADOS}

Las especies arbóreas encontradas en el parque Fuerza Aérea del Perú y la avenida Los Paracas son el eucalipto, la meliá, la ponciana, el molle costeño, el ficus, el tulipán africano y la palmera excelsa. De dicho grupo, las especies arbóreas que tienen mayor radio de copa son la ponciana, la meliá y el molle costeño, los cuales tienen disponibilidad de ofrecer mayor porcentaje de sombra en el espacio de la avenida y el parque.

Asimismo, se observó que las especies arbóreas identificadas como las más altas en la avenida y el parque son el tulipán africano, el eucalipto y la ponciana. Los árboles existentes son de porte mediano y pequeño.

Cabe anotar que la mayoría de los árboles de la zona de estudio son poncianas, una especie caducifolia. En cuanto a las restricciones físicas del parque, se consideraron las características morfológicas del suelo urbano y el equipamiento molesto, como postes, cables, entre otros.

Debido al estado de consolidación de la trama urbana, el suelo no posee impermeabilidad, debido a la presencia del asfalto de las vías vehiculares, del concreto de las veredas y las construcciones de viviendas multifamiliares. Los retiros de las calles son utilizados como estacionamientos o son reemplazados por rampas para el acceso vehicular a las edificaciones. Los parques son los principales componentes permeables en la zona de estudio. Por esta situación, la distribución de los árboles es aleatoria a lo largo de la avenida Los Paracas (véase la figura 2).

La configuración física de la avenida tiene una proporción muy baja, considerándose una vía urbana abierta (véase la figura 3). El uso de las edificaciones es residencial, y estas varían entre 2 y 9 pisos, lo que muestra el cambio de las viviendas unifamiliares a multifamiliares. Para dicho cálculo no se consideró la presencia de los árboles urbanos, debido a que no representan un borde significativo en los tramos de las calles. 
Figura 2

Presencia de árboles existentes

en la avenida Los Paracas y el parque Fuerza Aérea del Perú

Elaboración propia

Figura 3

Vía abierta: la

avenida Los

Paracas

Elaboración propia
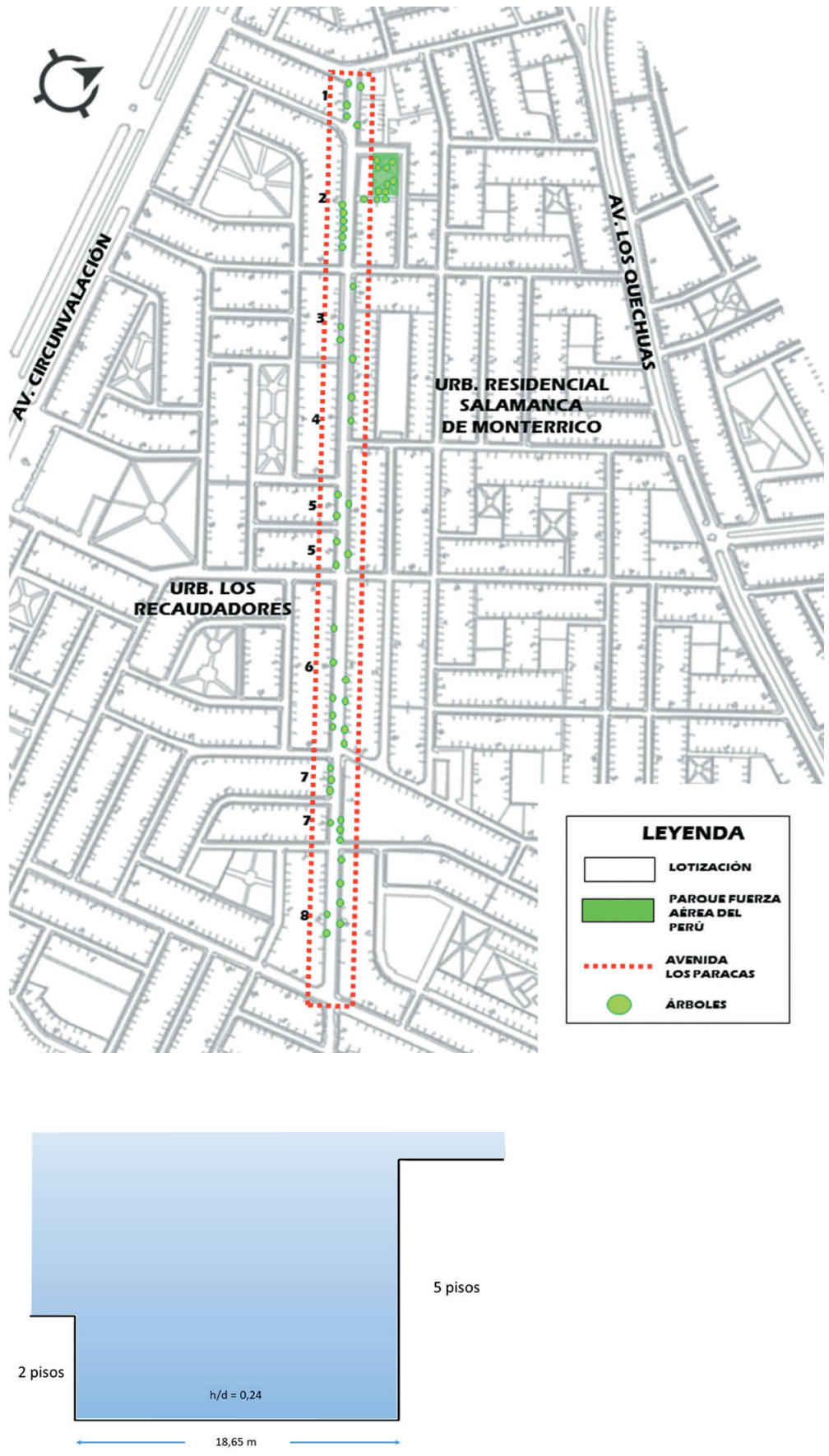
La configuración física del parque Fuerza Aérea del Perú, así como la altura de las edificaciones de la avenida Los Paracas y la dotación del arbolado, generan muy pocas zonas de sombra en los espacios de recorrido y de estancia, exponiendo gran parte de superficie al impacto solar. Por ello, se determinó que requiere un nivel de protección medio/alto.

La presencia de postes de alumbrado público y de energía, así como los cables eléctricos aéreos, básicamente se concentran en ambos lados de la calle, incluso cruzándose de lado a lado. También se presentan en el interior del parque, con un poste de señal wifi en su interior.

La percepción del verde urbano se definió en relación con la proporción de volumen verde. En comparación con las demás zonas del distrito, es la que cuenta con el mayor número de parques y jardines, por lo que se considera como pulmón de Ate. En este caso, se consideraron los tramos de la avenida Los Paracas, donde más del $10 \%$ del volumen verde se encuentra solo en un $25 \%$ de la longitud total de la avenida (véase la tabla 1 ). Dicho volumen verde está formado por árboles de mediano $(91,2 \%)$ y pequeño porte $(8,8 \%)$.

\begin{tabular}{|c|c|c|c|c|}
\hline $\begin{array}{c}\text { Tramos } \\
\text { de la avenida } \\
\text { Los Paracas }\end{array}$ & $\begin{array}{l}\text { Longitud } \\
\text { (m) }\end{array}$ & $\begin{array}{l}\text { Ancho } \\
(\mathrm{m})\end{array}$ & $\begin{array}{c}\Sigma \text { Volumen } \\
\text { de copa }\end{array}$ & $\begin{array}{c}\text { Porcentaje } \\
\text { de percepción } \\
\text { del verde urbano }\end{array}$ \\
\hline Cuadra 1 & 38 & 18,65 & 946,2 & 16,69 \\
\hline Cuadra 2 & 148,7 & 18,65 & 855,3 & 3,86 \\
\hline Cuadra 3 & 92 & 18,65 & 112,8 & 0,82 \\
\hline Cuadra 4 & 125 & 18,65 & 446,7 & 2,40 \\
\hline Cuadra 5 & 92 & 18,65 & 2834,9 & 20,65 \\
\hline Cuadra 6 & 182,6 & 18,65 & 1052,6 & 3,86 \\
\hline Cuadra 7 & 92 & 18,65 & 338,4 & 2,47 \\
\hline Cuadra 8 & 143,7 & 18,65 & 356,68 & 1,66 \\
\hline \multirow[t]{2}{*}{ Total } & 914 & & & \\
\hline & Objetivo mínimo & \multicolumn{2}{|c|}{ Objetivo deseable } & Objetivo alcanzado \\
\hline $\begin{array}{l}\text { Avenida } \\
\text { Los Paracas }\end{array}$ & $\begin{array}{l}>10 \% \text { del volumen } \\
\text { verde en más del } 50 \% \\
\text { de los tramos de calle }\end{array}$ & $\begin{array}{l}10 \\
\text { verd } \\
\text { de lo }\end{array}$ & $\begin{array}{l}\text { volumen } \\
\text { lás del } 75 \% \\
\text { os de calle }\end{array}$ & $\begin{array}{l}>10 \% \text { del volumen } \\
\text { verde en el } 25 \% \text { de } \\
\text { los tramos de calle }\end{array}$ \\
\hline
\end{tabular}


Por otro lado, los colores que se identifican en las flores grandes de las especies arbóreas son amarillo y anaranjado. En época de floración, las flores amarillas son las que predominan en los árboles de la zona de estudio. La ponciana, cuya flor es de color amarillo, es la especie arbórea con mayor cantidad de individuos en la avenida Los Paracas.

Siguiendo el principio de "crear colaboradores" de Trees \& Design Action Group (2012), se consideraron las acciones de la Municipalidad de Ate y de Salamanca Verde en torno al arbolado urbano. En cuanto a las acciones municipales, en el año 2014, se aprobó un régimen de intangibilidad, protección, conservación, defensa y mantenimiento de las áreas verdes de uso público, así como zonas de recreación pública en el distrito, como parte de su política ambiental. También se acordó promover acciones de sembrío con organizaciones vecinales, y desarrollo de programas de educación y sensibilización dirigidos a la población sobre el rol de las áreas verdes y sus beneficios. En el Plan de Desarrollo Local Concertado 2017-2021 (2017) del distrito de Ate, se tiene una visión alineada con la de Lima Metropolitana al 2021, "con armonía y respeto con el medio ambiente y el territorio; mejorando la calidad de vida [...] en un entorno moderno, saludable, seguro y responsable" (p. 19). Se ha realizado el mantenimiento en los parques de Salamanca a través del riego, corte de pasto y poda de árboles. Además, se espera que para el año 2030 se recuperen los espacios públicos para beneficio de los vecinos (pp. 16-17). Entre sus acciones estratégicas de primera prioridad, hasta el 2019, se contemplaron las siguientes:

- Recuperación de espacios públicos

- Implementación de un sistema de manejo de áreas verdes

- Desarrollo de espacios de recreación con enfoque ciudadano y ambiental

En el Programa Multinanual de Inversiones 2019-2021, entre las obras, se han considerado algunas relacionadas con el mejoramiento de parques en la zona 01 .

Por otro lado, en el año 2018, se formó la asociación ambiental Salamanca Verde que se define así:

Organización de vecinos y vecinas de las urbanizaciones de la zona 01 del Distrito de Ate que tiene por objeto recuperar y mejorar las áreas verdes de nuestras calles y parques, para contribuir con el equilibrio del medio ambiente, descontaminar el aire, reducir los efectos de la radiación solar y las emisiones de carbono que ocasiona el cambio climático; de esta forma aporta con la mejora de la calidad de vida de la población. (Salamanca Verde, s. f.)

A inicios del año 2019, Salamanca Verde tuvo su primera reunión con la Agencia Municipal de Salamanca, que expuso su plan de recuperación y arborización durante ese año: 
- Recuperación de la avenida Los Quechuas a través del sembrado de jardines con participación de los vecinos

- Recuperación de la avenida Los Paracas

- La meta para la zona 01-Salamanca fue plantar doscientos árboles

En febrero del 2019, se hizo una detención de la poda antitécnica de árboles en coordinación con la Agencia Municipal de Salamanca. Luego de un mes, Salamanca Verde alcanzó a la Municipalidad de Ate algunas recomendaciones para el proyecto de remodelación del parque Fuerza Aérea del Perú:

- Conservar y/o incrementar el número de árboles presentes en el parque.

- Cambiar palmeras por árboles adecuados para dotar de sombra, de acuerdo con las dimensiones del espacio terrestre y aéreo.

- Las jardineras perimetrales paralelas a las fachadas de las galerías podrían reubicarse a mayor distancia de estas para que los árboles plantados puedan tener una mejor cobertura del paseo y los lados a la galería. De esa forma, la copa no afectaría las fachadas de las galerías.

- Sobre las tensionadas en el medio del paseo, se recomendó reemplazarlas por estructuras de madera que permitan colocar enredaderas y así generar una cobertura vegetal que brinde sombra y confort.

- La zona de juego de niños debería estar rodeada de árboles que los protejan de los rayos solares en el verano.

En marzo del mismo año, se alcanzó a la Municipalidad de Ate un informe técnico sobre la poda de los árboles urbanos. Las recomendaciones brindadas fueron desatendidas, pues continuaron podando los árboles sin suficiente criterio. En los siguientes meses se identificaron casos de podas antitécnicas, en las que incluso los vecinos participaban (véase la figura 4). Las denuncias se realizaron ante la Municipalidad de Ate, exigiéndose una sanción y la reposición de árboles.

En abril del 2019, una comisión técnica de Salamanca Verde acudió a la primera reunión de la mesa de trabajo con la Municipalidad de Ate. Los puntos que se abordaron fueron los siguientes:

- Restitución y recuperación de áreas verdes: sustentada en el artículo 2 de la Ordenanza 525-MML.

- Adecuado mantenimiento de arbolado y áreas verdes e inventario de áreas verdes. Se mostró las fotos de las podas antitécnicas realizadas en los parques.

- Capacitación al personal de parques y jardines. 
Figura 4.

Aviso sobre las denuncias de las malas prácticas de

poda

Fuente: Reproducido de Salamanca Verde

(2019a)

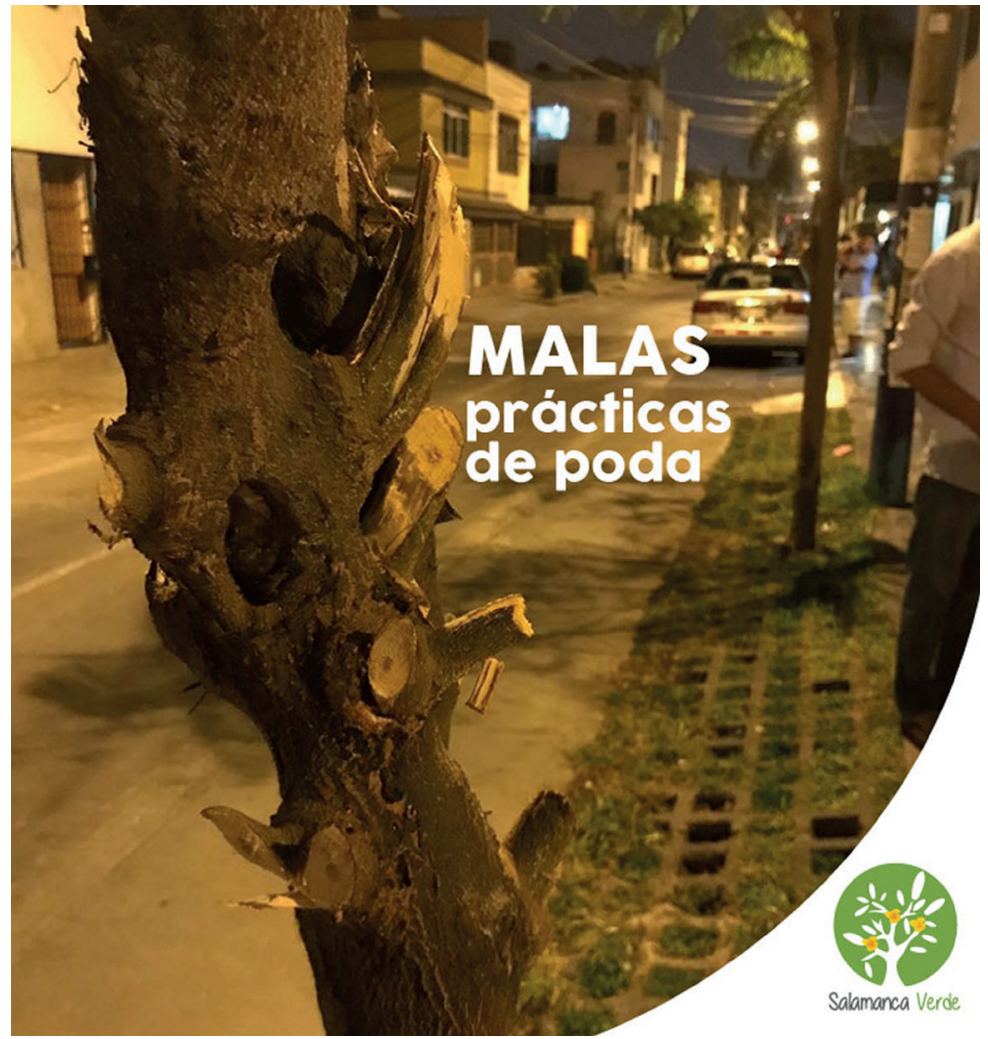

- Relación con las empresas constructoras: necesidad de una ordenanza de protección de las especies vegetales y arbóreas en el espacio del retiro municipal, bermas, pasajes. Ordenanza que fije un porcentaje equivalente entre espacios verdes y área construida.

- Agricultura urbana: promoción de la Ordenanza municipal 342-MDA, Programa "Techo Verde".

- Sensibilización y conciencia ambiental: formación de ciudadanos climáticamente inteligentes, desde la primera infancia.

- Potenciación de los viveros municipales.

- Acuerdo de que cada futura poda será informada para coordinar.

En septiembre del 2019, se presentó un memorial a la Municipalidad de Ate en el que se solicitaba detener las podas antitécnicas y la tala indiscriminada, la protección del arbolado urbano y del espacio público, la elaboración del inventario distrital de áreas verdes y del arbolado urbano, y el plan distrital de áreas verdes. Sin embargo, hasta la fecha no se ha recibido ninguna respuesta. 
Ahora la Asociación Peruana de Ecosistemas Urbanos y Arboricultura (APEUA) se ha sumado a esta labor prestando su apoyo técnico.

Tras un año sin manifestación de información alguna por parte de la Municipalidad de Ate respecto a la remodelación del parque Fuerza Aérea del Perú, en marzo del 2020 se supervisaron in situ las obras de remodelación del parque recién iniciadas, cuyo alcance consiste en el cambio de sus pisos y veredas. No se hacía referencia a la plantación ni al retiro de árboles, en relación con lo que se había recomendado: plantar nuevos árboles que dotaran de sombra a los espacios de recorrido y estancia del parque. Sin embargo, el proyecto quedó paralizado a causa de las medidas de aislamiento e inmovilización social por la pandemia del COVID-19. Salamanca Verde ha dejado expresa su preocupación por el alejamiento de sus actividades, debido a la coyuntura actual. Se espera que, superada dicha situación, se puedan retomar las actividades y cumplir con los objetivos trazados.

\section{CONCLUSIONES}

Después del análisis de los resultados, se identificó que la mayoría de los árboles urbanos existentes tienen un mayor radio de copa, lo que les permite proporcionar grandes zonas de sombra. Más del $50 \%$ de los árboles son poncianas y poseen un radio de copa de entre 2,5 y $6 \mathrm{~m}$, aunque es una especie arbórea caducifolia.

El número de árboles es insuficiente para brindar protección solar al parque y a la avenida. Las alturas de las edificaciones no son suficientes para producir sombras. La superficie de la avenida y del parque está desprovista de cualquier tipo de cobertura y expuesta a las inclemencias del clima durante el año. Por ello, es necesario aumentar las zonas de sombra para una mayor confortabilidad para los usuarios cuando usan el espacio público. De acuerdo con su configuración urbana abierta, la ubicación de árboles debería ser de entre 0 y $1 \mathrm{~m}$ de separación entre copas.

Los árboles existentes, además, son insuficientes para controlar el espacio abierto que genera el ancho de la avenida y el parque en el tejido urbano. Aunque se debe reconocer que dicha proporción urbana favorece la iluminación natural de las edificaciones de alrededor.

La presencia gris sobre la de verde va en aumento, ante las rampas y pavimentos que se encuentran en lugar de bermas. Esta situación, junto a la presencia del cableado aéreo eléctrico, reduce la permeabilización del suelo en la zona Salamanca.

La oportunidad de contar con árboles permite crear un paisaje urbano visual verde; sin embargo, el número de árboles es insuficiente para generar una buena presencia de verde en el espacio público. Esto plantea un desequilibrio de la relación gris-verde en la zona. Solo las cuadras 1 y 5 de la avenida cuentan 
con el número suficiente de árboles para obtener una adecuada presencia de verde. Se debe considerar que esta percepción está en relación con las distancias de las cuadras, y las dos señaladas anteriormente son algunas de las más cortas de la avenida Los Paracas. Cabe señalar que la ponciana, especie de mayor presencia en la zona de estudio, al poseer flores de color amarillo, tiene un gran valor de atractivo.

Además, se determinó que la Municipalidad de Ate tiene un manejo parcialmente sostenible de los parques vecinales de la zona 01 . El manejo que efectúa la municipalidad tiene como finalidad mejorar la calidad ambiental $\mathrm{y}$ de salud de los vecinos. A pesar de que existe una normatividad que ofrece un esquema general sobre la conservación, protección y manejo de las áreas verdes y parques con un enfoque sostenible, no es suficiente, porque también se debería reglamentar ciertos estándares de calidad en estos procesos para una adecuada gestión, evaluación, monitoreo y uso de los espacios públicos verdes. Por ello, las acciones realizadas por la municipalidad no son suficientes o carecen de un enfoque más holístico para el desarrollo de estos espacios en la urbe y mejorar la calidad de vida de los pobladores. El manejo de los parques en la zona de estudio no considera los costos de mantenimiento ni la valorización económica que tendría el terreno con una adecuada planificación, diseño, ejecución y monitoreo de parques. El tema debería verse de manera transdisciplinar, con un equipo técnico formado por arquitectos, biólogos, urbanistas, ingenieros forestales, entre otros.

La existencia de una guía que acompañe el proceso de planificación, diseño y manejo de áreas verdes como parques, bermas y jardines es esencial porque complementaría el marco normativo existente y, aunque no garantice parques habitables para la ciudad, supone una base técnica para los instrumentos necesarios para poder ejecutar. La gestión municipal, en combinación con la participación ciudadana, es la clave para llevar adelante esta premisa. En otros países como el Reino Unido, ya mencionado, se han formado organizaciones entre especialistas y la sociedad civil para proponer guías y acciones para el mantenimiento de los parques como espacios vitales de sus ciudades y centros de concentración e interacción entre los habitantes.

El sembrado de árboles, no solo en la subzona 03-Salamanca, sino en todo el distrito de Ate, se efectúa por consideraciones ambientales como purificadores de aire y elementos atractivos para el paisaje urbano del espacio público. Sin embargo, no se consideran sus aportes como reguladores de temperatura y un buen emplazamiento en el espacio urbano favorecería la habitabilidad del espacio público. Los árboles, cuando crecen sanos, viven más y son capaces de generar una verdadera recuperación del ambiente. Su capacidad productiva aumenta considerablemente a medida que crecen.

Debe asegurarse que tanto la siembra como el mantenimiento sean realizados por personas altamente capacitadas o supervisadas, seguidos de un monitoreo para evitar errores como daños a las raíces, la poda de forma inapropiada o la plantación de un árbol demasiado profundo o alto. En algunas situaciones será apropiado garantizar mediante accesorios la protección de los árboles jóvenes 
sin socavar la calidad urbana de su entorno. Hacer que estos accesorios de protección se vean atractivos ayuda a inspirar conciencia y limitar el vandalismo. Pero no se puede dejar sin mantenimiento adecuado a tales accesorios; de lo contrario, se pueden volver perjudiciales para el crecimiento del árbol.

Es recomendable definir estándares de suelo permeable para la plantación de árboles en los espacios públicos como calles y parques, como cuestión política y condición de planificación para la gestión de proyectos privados de construcción.

Se debe seguir investigando, estudiando los diferentes elementos que intervienen en estos procesos para mejorar las futuras acciones que se emprenderán para elevar la calidad de los parques y contribuir al desarrollo de comunidades sostenibles, como lo plantea la ONU entre sus Objetivos de Desarrollo Sostenible (Daniel, 2019). La información obtenida en esta investigación puede tomarse como punto de partida para su seguimiento de intervención en las demás áreas verdes de Salamanca, otras subzonas de la zona 01 del distrito de Ate o que tengan similares características físicas y de atractivo urbano. Lo que se pretende mostrar en este trabajo es que existe voluntad; existen ciudadanos preocupados por el cuidado, conservación y mantenimiento de su ambiente y que en conjunto se puede trabajar en beneficio de este.

Si bien la formación de grupos comunitarios requiere tiempo, la recompensa va más allá de los recursos corporativos que se puedan lograr. También cabe anotar la limitación de acción de este tipo de organizaciones civiles, muchas veces causada por el propio gobierno local y distrital que, en ocasiones, debido a la desidia u otros intereses mal priorizados, no permite enfatizar la vida en general como fin supremo. La arboricultura urbana no solo se trata de árboles, sino también de personas, porque mantener árboles urbanos sanos requiere contacto y apoyo de la comunidad. Eso es tener una visión más allá de la típica sostenibilidad: eso es tener un enfoque biocéntrico, donde la vida es el objetivo supremo.

Entonces, ¿Salamanca es una zona verde con suficiente presencia de verde? Se diría que Salamanca es una zona que está perdiendo su presencia de verde. Como parte del distrito de Ate, es una zona urbana consolidada en lo gris, pero aún es una zona urbana en proceso de consolidación en lo que concierne a lo verde y lo sostenible. Sin embargo, en la zona se puede hallar manos con voluntad y aún árboles por cuidar. "Power is in numbers" (Trees \& Design Action Group, 2012, p. 56). 


\section{REFERENCIAS}

Agencia de Ecología Urbana de Barcelona. (2011). Certificación del Urbanismo Ecosistémico. Edición del Autor.

CARTIF. (17 de septiembre del 2018). El proyecto europeo Urban GreenUp ofrece un catálogo de soluciones basadas en la naturaleza [Nota de prensa]. Construible.es. https://www.construible.es/2018/09/17/proyecto-europeourban-greenup-ofrece-catalogo-soluciones-basadas-naturaleza

Daniel, K. (2019). Objetivo 11 - Las ciudades desempeñarán un papel importante en la consecución de los Objetivos de Desarrollo Sostenible [Crónica $\mathrm{ONU}$. Naciones Unidas. https://www.un.org/es/chronicle/article/objetivo11-las-ciudades-desempenaran-un-papel-importante-en-la-consecucionde-los-objetivos-de

Del Caz-Enjuto, M. R. (2017). El papel de la vegetación en la mejora del entorno de los edificios en los procesos de regeneración urbana: estudio de caso. Urbano, 20(35), 102-113.

Galvez Nieto, A. (2019). Selección sostenible de árboles urbanos para beneficiar la habitabilidad del espacio público vecinal. Caso: parque Arróspide, Ate. Año 2018 [Tesis de maestría; Universidad Ricardo Palma]. file:///C:/Users/User/ Downloads/T030_42103288_T\%20\%20\%20GALVEZ\%20NIETO\%20 ALEXANDER\%20JUNIOR'S.pdf

García Estrada, E., De Hoyos Martínez, J. E., y Ávila Akerberg, V. D. (2020). Metabolismo ambiental. Cuidado y conservación de las áreas verdes urbanas. Contexto. Revista de la Facultad de Arquitectura de la Universidad Autónoma de Nuevo León, 14(20). https://doi.org/10.29105/ contexto14.20-5.

Hansmann, R., Whitehead, I., Krajter, S., Zivojinovic, I., Stojanovska, M., Jones, N., Bernasconi, A., Benamar, S., Lelieveld, C., y Barstad, J. (2016). Partnerships for Urban Forestry and Green Infrastructure Delivering Services to People and the Environment: A Review on What They Are and Aim to Achieve. SEEFOR. South-East European Forestry, 7(1), 9-19.

Mínguez, E., Martí, P., Vera, M., y Meseguer, D. (2013). Claves para proyectar espacios públicos confortables. Indicador del confort en el espacio público. Enrique Mínguez Arquitectos.

Ordenanza 1852 [Municipalidad Metropolitana de Lima]. Ordenanza para la conservación y gestión de áreas verdes en la provincia de Lima. 23 de diciembre del 2014. http://smia.munlima.gob.pe/uploads/ documento/793d8fbb0c8e70f5.pdf

Municipalidad de Ate. (2003). Plan Integral de Desarrollo del Distrito de Ate 2003-2015. Zona $01 . \quad$ https://docplayer.es/80872347-Municipalidad-distrital-de-ateplan-integral-de-desarrollo-del-distrito-de-ate-zona-01-alcalde-oscarbenavides-majino.html 
Municipalidad de Ate. (2017). Plan de Desarrollo Local Concertado 2017-2021 del Distrito de Ate. http://www.muniate.gob.pe/ate/files/documentosPlanea mientoOrganizacion/POLITICAS_PUBLICAS/PLANDESARROLLOLOCAL CONCERTAD02017-2021_ATE.PDF

Pérez-Medina, S., y López-Falfán, I. (2015). Áreas verdes y arbolado en Mérida, Yucatán. Hacia una sostenibilidad urbana. Economía, Sociedad y Territorio, XV(47), 1-33. http://www.scielo.org.mx/pdf/est/v15n47/v15n47a2.pdf

Restrepo, H., Moreno, F., y Hoyos, C. (2015). Incidencia del deterioro progresivo del arbolado urbano en el valle de Aburrá, Colombia. Colombia Forestal, 18(2), 225-240.

Reyna Valencia, C. (2017). Gestión ambiental participativa. El caso de un área de protección de recursos naturales en Valle de Bravo [Tesis de maestría; Universidad Autónoma del Estado de México]. http://ri.uaemex. $\mathrm{mx} /$ bitstream/handle/20.500.11799/94952/4.\%20MAESTRIA\%20 CARLOS\%202017.pdf?sequence=3\&isAllowed=y

Ruiz, M., Correa, E., y Cantón, M. (2016). Diseño eficiente de parques en ciudades de zonas áridas. Confort térmico y clima urbano. En Acta del I Encuentro Nacional sobre Ciudad, Arquitectura y Construcción Sustentable (pp. 105-116). Universidad Nacional de La Plata.

Salamanca Verde. (s. f.). Información [Página de Facebook]. Facebook. Recuperado el 9 de junio del 2020 de https://www.facebook.com/groups/29428937789 1887/permalink/533471837306972/

Salamanca Verde. (8 de febrero del 2019a). Malas prácticas de poda [Imagen adjunta]. Facebook. https://www.facebook.com/groups/294289377891887/ posts/731379494182871/

Salamanca Verde. (12 de febrero del 2019b). Nuestra reunión de los sábados se trasladó a la Agencia Municipal de Salamanca. Facebook. https://www. facebook.com/groups/294289377891887/posts/313143022673189

Shigyo, V. (2017). Introducción a la arquitectura sostenible. Universidad Ricardo Palma, Escuela de Posgrado.

Trees \& Design Action Group. (2012). Trees in the Townscape. A Guide for Decision Makers. https://www.tdag.org.uk/uploads/4/2/8/0/4280686/tdag_treestowns cape2021.pdf

Valdivia-Loro, A. (2019). Evaluación del índice de sostenibilidad urbana. Aplicación para Lima Metropolitana. Bitácora Urbano Territorial, 29(3),135-144 .

Villazán, A., y Huertas, R. (2019). El proyecto Urban GreenUp en Valladolid: estrategia aplicada de renaturalización urbana. En Renaturalización de la ciudad (pp. 288-297). Diputació de Barcelona. 
